\title{
Indice infiammatorio della dieta: correlazioni con la densità ossea minerale e il rischio di fratture in donne dopo la menopausa: risultati dallo studio WHI
}

\author{
S. Migliaccio ${ }^{1}$
}

Pubblicato online: 22 febbraio 2017

(C) Springer International Publishing AG 2017

\section{Commento a:}

Dietary inflammatory index, bone mineral density and risk of fracture in postmenopausal women: results from the Women's Health Initiative.

T. Orchard, V. Yildiz, S.E. Steck, J.R. Hébert, Y. Ma, J.A. Cauley, W. Li, Y. Mossavar-Rahmani, K.C. Johnson, M. Sattari, M. LeBoff,

J. Wactawski-Wende, R.D. Jackson.

J Bone Miner Res (2016) doi:10.1002/jbmr.3070

Molteplici studi suggeriscono una correlazione tra un incremento dello stato infiammatorio del soggetto e un aumentato rischio di sviluppare alterazioni della densità minerale ossea e fratture da fragilità. In questo studio gli autori hanno valutato una potenziale correlazione tra l'indice infiammatorio della dieta e un aumentato rischio di fratture in donne dopo la menopausa.

Vari studi suggeriscono che la perdita di massa ossea e il rischio di fratture siano associati a un'infiammazione cronica, potenzialmente modificabile dalla dieta. L'obiettivo primario dell'analisi effettuata dagli autori era valutare l'associazione tra l'indice infiammatorio della dieta (DIITM), una misura del potenziale infiammatorio, con il rischio di frattura utilizzando i dati longitudinali ottenuti dallo studio osservazionale Women's Health Initiative (WHI). Sono state anche valutate le correlazioni tra densità minerale ossea (BMD) e punteggi DII. I punteggi DII sono stati calcolati sui questionari di frequenza alimentare (FFQ) completati da 160.191 partecipanti (età media 63 anni), senza pregressa storia di frattura di femore. Le fratture sono state segnalate almeno una volta all'anno e confermate dalle cartelle cliniche. Il rischio di sviluppare fratture è stato calcolato utilizzando un modello di analisi di rischio proporzionale multivariata stratificata per età e etnia. La valutazione delle variazioni di $\mathrm{BMD}$, misurate con DXA rispetto al basale (al terzo e sesto anno), è stata effettuata analizzando i quartili $(\mathrm{Q} 1$ = dieta meno infiammatoria) dei punteggi DII basali. Il punteggio medio DII è risultato migliorato nel corso di tre anni $(p<0,01)$, ma il cambiamento non è risultato associato con il rischio di fratture. Il punteggio basale DII si associava solo al rischio di frattura di femore in donne caucasiche (HR Q4: 1,48; 95\% CI: 1,09-2,01; $p=0,01$ ), mentre non si riscontrava un'associazione significativa in donne caucasiche di età superiore ai 63 anni. Le donne con punteggio DII più basso avevano una minore perdita di densità minerale ossea a livello femorale $(p=0,01)$, nonostante la bassa densità minerale ossea femorale iniziale, rispetto a donne con punteggi di DII più elevati. In conclusione, un modello alimentare meno infiammatorio risulta associato con una minore perdita di BMD in donne dopo la menopausa. Una dieta con un più alto indice infiammatorio risulta associato a un aumento del rischio di frattura dell'anca solo in donne caucasiche di età inferiore ai 63 anni.
S. Migliaccio

silvia.migliaccio@uniroma1.it

1 Department of Movement, Human and Health Sciences, University Foro Italico of Rome, Piazza Lauro De Bosis 6, Roma, Italia 